\title{
Role of Non-muscle Myosin Light Chain Kinase in Neutrophil- mediated Intestinal Barrier Dysfunction During Thermal Injury
}

\author{
Mingzhang Guo ${ }^{3}$, Sarah Y. Yuan², Chongxiu Sun ${ }^{3}$, Bert J. Frederich ${ }^{3}$, Qiang Shen ${ }^{3}$, \\ Danielle L. McLean ${ }^{2}$, and Mack H. Wu ${ }^{1,2}$ \\ 1'James A Haley VA Hospital, Tampa, Florida 33612 \\ 2University of South Florida Morsani College of Medicine, Tampa, Florida 33612 \\ ${ }^{3}$ University of California Davis School of Medicine, Sacramento, CA 95817
}

\begin{abstract}
Neutrophils and non-muscle myosin light chain kinase (nmMLCK) have been implicated in intestinal microvascular leakage and mucosal hyperpermeability in inflammation and trauma. The aim of this study was to characterize the role of nmMLCK in neutrophil-dependent gut barrier dysfunction following thermal injury, a common form of trauma that typically induces inflammation in multiple organs. Histopathological examination of the small intestine in mice after a full-thickness burn revealed morphological evidence of mucosa inflammation characterized by neutrophil infiltration into the lamina propria, epithelial contraction, and narrow villi with blunt brush borders and loss of goblet cells. Compared to their wild-type counterparts, nmMLCK-/mice displayed diminished morphological abnormalities. Likewise, intravital microscopic studies showed significant leukocyte adhesion in intestinal microvessels post-burn, a response that was blunted in the absence of nmMLCK. Functionally, thermal injury significantly increased the gut lumen-to-blood transport of FITC-dextran $(4 \mathrm{kD})$, and this hyperpermeability was attenuated by either neutrophil depletion or nmMLCK deficiency. Consistent with the in vivo observations, in vitro assays with Caco-2 epithelial cell monolayers revealed a decrease in transcellular electric resistance coupled with myosin light chain phosphorylation, actomyosin ring condensation, and claudin-1 internalization upon stimulation with fMLP-activated neutrophils. Pretreatment of the cells with the MLCK inhibitor ML-7 prevented the tight junction responses. Taken together, the results suggest that nmMLCK plays an important role in neutrophil-dependent intestinal barrier dysfunction during inflammatory injury.
\end{abstract}

\section{Keywords}

Myosin light chain kinase; neutrophil; intestinal barrier dysfunction; signal transduction

\section{INTRODUCTION}

The intestinal epithelium forms a critical interface between the interstitium and the harsh luminal environment consisting of acid, enzymes, microbes, and toxins (1). The gut epithelium functionally serves as a semi-permeable barrier to control selective transport and absorption of nutrients, electrolytes, and water and to protect the internal milieu from exposure to a hostile luminal environment. An intact gut barrier is of vital importance to normal intestinal physiological function and host homeostasis. Disruption of intestinal 
barrier integrity represents both a salient feature and a vital pathogenic player of many important gastrointestinal disorders that occur during inflammatory and traumatic injuries $(1,2)$.

Severe burn injury often induces systemic inflammatory response syndrome (SIRS), characterized by microvascular circulatory disturbance of splanchnic organs distant from the burn site, acute respiratory syndrome and, multiple organ failure (MOF) (2-4). Systemic inflammation leads to leukocyte activation and transvascular migration, microvascular endothelial hyperpermeability, and intestinal epithelial barrier dysfunction (2, 5-8), all key contributors to the development of septic complications and MOF. Numerous burn-related deaths are associated with complications of infection or sepsis, chiefly due to burn wound infections, nosocomial infections, and pneumonia (9). Loss of gut barrier integrity, occurring secondarily to cutaneous burn injury, has been recognized as a key player in septic pathogenesis (2-4). Thermal injury also induces splanchnic vasoconstriction resulting in decreased intestinal blood flow and impaired tissue perfusion. Increased microvascular plasma leakage and excessive interstitial fluid accumulation trigger tissue ischemiareperfusion and hypoxic injuries that, in turn, exacerbate mucosal hyperpermeability, bacterial translocation and endotoxin absorption from the gut $(2,10,11)$. Notably, the gut damage begins shortly after burn $(8,12-14)$, and the activation and transmigration of polymorphonuclear leukocytes (PMNs) is directly associated with loss of gut barrier function $(11,15,16)$. Activated PMNs are capable of triggering the breakdown of both microvascular endothelium and intestinal epithelial mucosa through mechanisms involving the activation of intracellular protein kinases and molecular modifications of the structural proteins at cell-cell adherens junctions and/or tight junctions (1, 17-19). Other mechanisms leading to mucosal barrier damage post-burn include the production and release of proinflammatory cytokines, increased formation of reactive oxygen species (ROS) and reactive nitrogen species, and impaired host immune defense $(1,2,4)$. To date, the molecular mechanisms underlying PMN-mediated intestinal barrier dysfunction in burns remain incompletely defined. A deeper understanding of this process will likely provide new insights to aid in the design and development of more effective therapeutic strategies toward the treatment of burns and related inflammatory syndromes.

Gut barrier function largely relies on the integrity of the tight junctions (TJs) of intestinal epithelium. MLCK-mediated phosphorylation of myosin light chain (MLC) has emerged as an important regulatory event in intestinal $\mathrm{TJ}$ assembly/disassembly and paracellular permeability (1). MLCK is a $\mathrm{Ca}^{2+}$-calmodulin-dependent serine/threonine kinase that dynamically regulates actomyosin reorganization and cell contraction in response to various stimuli. The MLCK family is comprised of a non-muscle or long isoform $(210 \mathrm{kDa})$, a short smooth-muscle form $(108 \mathrm{kDa})$, and a telokin $(21 \mathrm{kDa})$ that lacks enzymatic activity. nmMLCK is predominantly expressed in endothelium, epithelial cells, and PMNs (1, 20-22), and has been implicated in the regulation of many pivotal cell functions. Indeed, MLCK activation plays a key role in microvascular hyperpermeability evoked by neutrophil stimulation and during thermal injury $(5,6,18,22)$. nmMLCK is essential to $\mathrm{Na}^{+}$-nutrient co-transport and TNFa-induced intestinal epithelial barrier loss and mucosal damage $(1,24$, 25). Genetic deletion of nmMLCK enhances both intestinal endothelial and epithelial barrier properties and confers protection against burn-induced vascular leakage (6) and T cell activation-mediated intestinal epithelial barrier dysfunction and diarrhea (26). Recently, $n m M L C K$ has been shown to mediate $\beta 2$ integrin activation and transendothelial migration of neutrophils (22).

Although nmMLCK is known to play an important role in the physiological and pathophysiological regulation of intestinal epithelial tight junctions and paracellular leak pathways, its functional implications in burn-induced intestinal barrier loss remain poorly 
understood. To extend our previous finding that nmMLCK activation mediates microvascular hyperpermeability during vascular inflammation $(5,6,18,22)$, we studied the direct impact of a full-thickness burn on neutrophil dynamics and recruitment, microvascular barrier function as well as intestinal epithelial barrier function. We combined morphological examination with both in vivo and in vitro functional assays. It was found that severe thermal injury caused intestinal barrier dysfunction and mucosal villous damage, which was largely prevented by either neutrophil depletion or genetic deletion of nmMLCK. Wild type mice exhibited significantly increased leukocyte recruitment in intestinal postcapillary venules and neutrophil infiltration into intestinal lamina propria. This response was diminished in nmMLCK-/- mice. Neutrophil stimulation led to intestinal epithelial cell contraction and monolayer barrier dysfunction, which were prevented by pharmacologic inhibition of MLCK. These results suggest that nmMLCK plays an important role in neutrophil-mediated intestinal barrier loss and mucosal damage during inflammatory injury. nmMLCK may represent a prominent target for therapeutic intervention in intestinal inflammatory disease and traumatic injury.

\section{MATERIALS AND METHODS}

\section{Cells and Reagents}

The human epithelial colon adenocarcinoma (Caco-2) cell line and Eagle's minimum essential medium (EMEM) were purchased from ATCC (Baltimore, MD). Phalloidin Alexa-488, Hoechst 33342, and anti-claudin-1 antibody were from Invitrogen (Carlsbad, CA). Antibodies to myosin light chain (MLC) and phosphorylated MLC (Thr18/Ser19) were from Cell Signaling (Beverly, MA). Other antibodies included anti- $\beta$-actin and TRITClabeled goat anti-rabbit IgG conjugate (Santa Cruz Biotechnology, Santa Cruz, CA), antiGr-1, RB6 (BioLegend, San Diego, CA), and anti-Ly-6G (BD Biosciences, San Jose, CA). Fluorescein isothiocyanate-dextran FD-4, acridine orange, N-formyl-methionyl-leucylphenylalanine (fMLP), hematoxylin, and eosin were from Sigma (St. Louis, MO). Compound ML-7 was purchased from Calbiochem (San Diego, CA).

\section{Animal Procedures}

This study was approved by the Institutional Animal Care and Use Committee of University of California at Davis and was performed in accordance with criteria outlined in the National Institutes of Health Guide for Care and Use of Laboratory Animals. Male mice at 15-25 weeks of age were anesthetized with an intramuscular injection of urethane at $1.75 \mathrm{~g} /$ $\mathrm{kg}$. The right jugular vein and carotid artery were cannulated for infusion of drugs or solutions and continuous monitoring of blood pressure, respectively. A $40 \%$ total body surface full-thickness burn was induced over the shaved dorsal and ventral skin, as described previously $(6,27)$. Briefly, an adjustable burn template that accounted for variation in body surface area was filled with boiling water, and placed on the dorsal and ventral skin for 10 seconds each. Control groups were subjected to the same procedure with water at $37^{\circ} \mathrm{C}$. After burn, animals were given a subcutaneous injection of lactated Ringer's Injection (Baxter Healthcare, Deerfield, IL) at $0.025 \mathrm{~mL} / \mathrm{g}$, according to the Parkland formula.

\section{Depletion of Neutrophils}

To deplete neutrophils in C57BL/6 mice, $200 \mathrm{mg}$ of monoclonal anti-Gr-1 IgG, RB6, was administered intraperitoneally $24 \mathrm{~h}$ prior to the burn experiment. Control mice were inoculated with an equal volume of PBS. On the day of the experiment, a drop of blood was collected from the retro-orbital plexus, and blood smears were examined for verification of neutrophil depletion. Percentages of specific leukocyte types were determined from the total number of cells counted. RB6-treated mice displayed $>90 \%$ reduction of peripheral blood neutrophils, which accounted for less than $5 \%$ of the circulating leukocytes. 


\section{Measurement of Intestinal Mucosal Permeability}

Intestinal paracellular permeability assay was performed as previously described $(8,14)$. Briefly, a midline laparotomy was performed on mice at $3 \mathrm{~h}$ after burn or sham treatment. A $5-\mathrm{cm}$ segment of the jejunum beginning at $5 \mathrm{~cm}$ distal to the ligament of Treitz was dissected and tied with suture at both of the lateral ends. Two hundred microliters of FITCdextran (FD-4, MW 4,300, $125 \mathrm{mg} / \mathrm{mL}$ ) in $0.1 \mathrm{M}$ phosphate-buffered saline (pH 7.2) was injected into the jejunum lumen. The intestine was returned to the abdominal cavity. After $30 \mathrm{~min}$, blood specimens were collected by retro-orbital bleeding, followed by immediate dilution in $50 \mathrm{mM}$ Tris solution with $150 \mathrm{mM} \mathrm{NaCl}$ and centrifugation at 3,000 $\mathrm{g}$ for $10 \mathrm{~min}$ at $4^{\circ} \mathrm{C}$. The plasma concentrations of FITC-dextran were determined based on a standard curve of reference FITC-dextran using a fluorescence spectrophotometer.

\section{Intravital Microscopy and Leukocyte-Endothelium Adhesion}

A midline laparotomy was performed, and a section of jejunum mesentery was exteriorized over an optical stage for microscopic observation. The exteriorized mesentery was constantly superfused with lactated Ringer's Injection at $37^{\circ} \mathrm{C}$. The mesenteric microcirculation was examined using a Nikon Eclipse E600FN Microscope equipped with a Photometrics Cascade 512F digital camera under a 10x working distance objective (Technical Instruments, Burlingame, CA). Leukocytes were labeled with acridine orange via intravenous infusion $\left(0.05 \mathrm{~mL} ; 1.65 \times 10^{-4} \mathrm{M}\right)(7)$. Postcapillary venules were selected for observation. Leukocyte rolling and adhesion were analyzed 1 and $2 \mathrm{~h}$ postburn in mesenteric postcapillary venules (15-25 $\mu \mathrm{m}$ in diameter). All image acquisition and analysis was performed using Simple PCI software. Digital fluorescent image sets were acquired over a 60 -s period to evaluate leukocyte dynamics. Leukocyte adherence to the endothelium was determined by variable speed playback of the digital image sets. A Leukocyte was defined as adherent if it remained stationary for $230 \mathrm{~s}$; leukocyte adherence is measured through the observation period and expressed as cell number per $100 \mu \mathrm{m}$ of vessel in length.

\section{Histology and Immunohistochemistry of Intestinal Mucosal Tissue}

Mouse intestinal segments (jejunum or ileum, $\sim 1 \mathrm{~cm}$ long) were harvested $4 \mathrm{~h}$ postburn, and fixed in tissue freezing medium O.C.T. compound (Sukura Finetek, Torrance, CA). Intestinal segments were sectioned using a Leica CM3050 S cryostat (Leica Microsystems, Bannockburn, IL) at $8 \mu \mathrm{m}$ thickness, mounted on slides, and fixed with $95 \%$ ethanol. Standard hematoxylin-eosin staining was performed to examine intestinal morphology. Histological gut injury was assessed (by a trained pathologist) using a pathological scoring system in a blinded manner. Specimens were scored for degree of gut injury on a scale from 0 to $4: 0=$ normal; no pathology; 1 = mild; focal epithelial edema; $2=$ moderate; epithelial disruption, loss of goblet cells, and partial shortening and diffuse necrosis of the villi; $3=$ severe, diffuse pathology of the villi; with evidence of severe neutrophil infiltration in the submucosa; 4 = major; widespread injury with massive neutrophil infiltration and hemorrhage as previously described (28). The length and width of intestinal villi from gut sections was measured using the image analysis software MetaMorph (Molecular Devices, Sunnyvale, CA). Neutrophils in intestine were immunostained using the ABC method. Briefly, fixed cryosections were incubated with $\mathrm{H}_{2} \mathrm{O}_{2}$ for inhibition of endogenous peroxidase, and blocked with normal rabbit serum. Cryosections were incubated for $90 \mathrm{~min}$ with an anti-Ly-6G/Gr-1 antibody. After washing, the sections were incubated with a biotinylated secondary conjugate, followed by incubation with $\mathrm{ABC}$ reagent and the substrate AEC (Vector Laboratories, Burlingame, CA) for color development. The sections were then counterstained with hematoxylin, mounted with glycerol gelatin, and examined by light microscopy. For scoring leukocyte infiltration in the lamina propria, the following scoring system was used: $0=0-5$ PMNs; $1=6-10$ PMNs, $2=11-20$ PMNs, $3=21$ PMNs, 
and above. Total scores were averaged from 5-10 random, high-power fields from each intestinal segment.

\section{Neutrophil Purification and Activation with fMLP}

Human neutrophils were isolated and purified from heparinized normal whole blood by onestep percoll density-gradient centrifugation. Neutrophil preparations were confirmed to be $\sim 95 \%$ pure. For activation, purified neutrophils in Hank's buffered salt solution at $5 \times 10^{7}$ cells/mL were incubated with $1 \mu \mathrm{M}$ fMLP for $15 \mathrm{~min}$ at $37^{\circ} \mathrm{C}$, and centrifuged at $400 \times \mathrm{g}$ for $5 \mathrm{~min}$ at room temperature. Neutrophils were immediately suspended in serum-free EMEM, and added at a final concentration of $5 \times 10^{6}$ cells $/ \mathrm{mL}$ to cultured Caco- 2 cells in dishes or wells of gold electrode arrays for stimulation.

\section{Measurement of Transepithelial Electrical Resistance (TER)}

The intestinal epithelial cell barrier property was evaluated by measuring transepithelial electrical resistance (TER) with an electric cell-substrate impedance sensing (ECIS) system. Human epithelial colon adenocarcinoma cell (Caco-2) monolayers spontaneously differentiate and display morphological and functional characteristics of mature smallintestinal enterocytes, and thus have been well established as an in vitro model for intestinal epithelial permeability (29). Briefly, Caco-2 cells were seeded onto gold electrode arrays (Applied Biophysics, Troy, NY) at 100,000 cells/well and grown for $10-14$ days at $37^{\circ} \mathrm{C}$, with medium changed every other day. With culture medium serving as the electrode, epithelial cell barrier function was dynamically measured by determining the electrical impedance of a cell-covered electrode. A 1-V, 4,000-Hz alternating current signal was supplied through a 1-M resistor to approximate a constant-current source. The in-phase voltage (proportional to resistance) and out-of-phase voltage (proportional to capacitive resistance) were measured and analyzed with the ECMS 1.0 software (CET, Coralville, IA). Epithelial barrier function was expressed as TER normalized to baseline.

\section{Western Blotting}

Caco- 2 cells were grown to confluence in EMEM with $20 \%$ fetal bovine serum (FBS). Cells were starved in EMEM with $0.5 \%$ FBS for $4 \mathrm{~h}$ prior to treatment. After neutrophil or vehicle stimulation, cells were lysed in a RIPA buffer containing 1\% NP-40, phosphatase inhibitors ( $1 \mathrm{mM}$ sodium orthovanadate, $50 \mathrm{mM} \mathrm{NaF}, 2 \mathrm{mM} \beta$-glycerol phosphate, $2 \mathrm{mM}$ sodium pyrosophosphate), $1 \mathrm{mM}$ dithiothreitol, $1 \mathrm{mM}$ EDTA, and a cocktail of protease inhibitors. Cell lysates were clarified by centrifugation at $13,000 \times \mathrm{g}$ for $20 \mathrm{~min}$, and proteins in the supernatants were separated by SDS-PAGE, transferred to PVDF membrane, and blotted with antibodies to MLC, phospho-MLC or $\beta$-actin. The membrane was incubated with a corresponding HRP-labeled secondary conjugate, and the immunoreactive bands were detected using the Pico Supersignal chemiluminescent substrate (Pierce, Rockford, IL). Images of the blots were acquired by reflectance scanning densitometry, and band intensity quantified using Image $\mathrm{J}$ software.

\section{Immunofluorescence Microscopy}

Caco-2 cells were grown on Lab-Tek chamber slides (Nalge Nunc, Rochester, NY) in EMEM with $20 \%$ FBS. After reaching $100 \%$ confluence, Caco-2 cell monolayers were rinsed once with PBS and refilled with EMEM. Cells were pretreated with $1 \mu \mathrm{M} \mathrm{ML}-7$ or vehicle for $30 \mathrm{~min}$, and incubated at $37^{\circ} \mathrm{C}$ for $8 \mathrm{~h}$ with fMLP-activated neutrophils at a final concentration of $5 \times 10^{6}$ cells $/ \mathrm{mL}$. Caco- 2 monolayers were then washed with PBS, fixed with $3.7 \%$ paraformaldehyde for $10 \mathrm{~min}$, and permeabilized with $0.1 \%$ Triton $\mathrm{X}-100$ for 5 min at room temperature. After blocking with $1 \%$ BSA in PBS, cells were incubated for 30 min with phalloidin Alexa-488 for F-actin staining. For claudin-1 labeling, Caco-2 cells 
were incubated for $1 \mathrm{~h}$ sequentially with an anti-claudin-1 antibody and TRITC-labeled goat anti-rabbit conjugate. After washing, cells were incubated with Hoechst 33342 for 10 min, and examined through an Axiovert 200M fluorescent microscope equipped with an AxioCam MR camera (Carl Zeiss, Thornwood, NY). Digital images were acquired with Zeiss Axiovision 4.0 software.

\section{Data Analyses}

For in vivo experiments, $n$ represents the number of animals studied. For in vitro cell experiments, $n$ is given as the number of dishes or wells of cultured Caco-2 cells. Data are expressed as mean \pm S.E.M. For statistical analysis, paired Student $t$ test was used for selfcomparison, and ANOVA followed by Turkey HSD was used to evaluate the significance of inter-group differences. Significance was accepted at the level of $P<0.05$.

\section{RESULTS}

\section{Burn-induced Disruption of Intestinal Barrier Function}

Histopathological examination of mouse small intestinal specimens revealed that WT mice displayed marked villous shrinkage, and blunting and sloughing in the jejunum (Fig. 1A) and ileum (data not shown) after thermal injury. Burned WT mice exhibited a significant decrease in the length $(83.77 \pm 0.03 \%$ of sham $)$ and width $(60.99 \pm 0.03 \%$ of sham $)$ of small intestinal villi when compared with sham animals, suggesting the development of villous atrophy. Thermal injury induced intermediate gut injury $4 \mathrm{~h}$ post-burn, based on pathological scoring data (Fig. 1C). In contrast, nmMLCK-/- animals subjected to thermal injury exhibited normal histological appearance of small intestinal villi, with little or minimal injury (Fig. 1A, 1C). This finding was supported by the largely unaffected jejunum villi in burn nmMLCK-/- mice, which demonstrated unaltered length $(94.67 \pm 0.04 \%$ of sham) and width (97.58 $\pm 0.05 \%$ of sham). Immunohistochemistry showed increased infiltration of labeled neutrophils into the lamina propria of the small intestine in burned WT mice when compared to control mice (Fig. 1B, 1C). Notably, this leukocyte infiltration was largely abrogated in nmMLCK-/- mice subjected to the same thermal injury (Fig. 1B, 1C).

\section{Impact of Burn Injury on Intestinal Leukocyte Adhesion}

Leukocyte adhesion to mesenteric postcapillary venules was evaluated in both nmMLCK-/and WT mice at $1 \mathrm{~h}, 2 \mathrm{~h}$, and $3 \mathrm{~h}$ post burn or sham treatment. All venules chosen for the leukocyte adhesion study were comparable in shear rate and diameter $(16-25 \mu \mathrm{m})$. At 1 hour after treatment, burned WT mice exhibited significantly greater leukocyte adhesion in postcapillary venules when compared with sham-treated controls (Fig. 2B). Whilst there was a significant increase in leukocyte adhesion in nmMLCK-/- mice subjected to thermal injury as compared to sham mice, the magnitude of this adhesion was significantly lower than that observed in burned WT mice and was equivalent to that in sham-treated WT mice (Fig. 2B). Likewise, leukocyte adhesion remained significantly elevated in burned WT mice $2 \mathrm{~h}$ (Fig. 2C) and $3 \mathrm{~h}$ (data not shown) post-burn when compared with control animals, with no significant difference in leukocyte adhesion between burned nmMLCK-/- mice and shamtreated WT mice at these time points. Interestingly, nmMLCK-/-mice exhibited significantly lower leukocyte adhesion than WT mice under normal physiological conditions.

\section{Burn-induced Intestinal Paracellular Hyperpermeability}

As shown in Fig. 3, a 40\% scald burn led to significantly increased intestinal mucosal permeability to FITC-dextran FD-4 (MW 4,400) in WT mice, and this hyperpermeability response was diminished in nmMLCK-/- mice. Notably, intestinal hyperpermeability postburn was prevented by neutrophil depletion in WT animals (Fig. 3). There was no difference 
in basal intestinal permeability among sham-treated WT, nmMLCK-/-, and neutrophildepleted WT mice.

\section{Neutrophil-induced Intestinal Epithelial Barrier Dysfunction}

Intestinal epithelial barrier function was assessed by measurement of transepithelial electrical resistance (TER) using an ECIS system. Incubation of Caco-2 cell monolayers with fMLP-activated neutrophils induced a significant decrease in TER (Fig. 4A,B), indicative of epithelial barrier dysfunction. Pretreatment of Caco-2 cells with ML-7, a selective MLCK inhibitor, abolished neutrophil-induced intestinal epithelial barrier dysfunction (Fig. 4A,B). ML-7 alone caused only minimal changes in TER in Caco-2 cell monolayers.

\section{Neutrophil-induced MLC Phosphorylation}

In Caco-2 cells, fMLP-activated neutrophils induced a significant increase in MLC phosphorylation at threonine 18/serine 19, with no change in total MLC levels evident before or after treatment (Fig. 5). The MLC phosphorylation response was significantly attenuated in neutrophil-activated Caco-2 cells in the presence of ML-7, a selective MLCK inhibitor (Fig. 5).

\section{Disruption of Epithelial Tight Junctions and Claudin-1 Re-distribution}

Immunofluorescence microscopy revealed that normal intestinal epithelial monolayers exhibited intact intercellular junctions, at which the tight junction molecule claudin-1 colocalized with peri-junctional F-actin (the merge field, Fig. 6A), forming a characteristic ring-like structure. Stimulation of Caco-2 cell monolayers with fMLP-activated neutrophils provoked sequestration and internalization of claudin- 1 from intercellular junctions, coupled with intercellular gap formation (Fig. 6B). Likewise, F-actin stress fiber labeling was greatly increased following neutrophil stimulation, and exhibited an abnormal diffusive distribution pattern along cell-cell contacts. The co-localization of claudin-1 with F-actin was largely disrupted (Fig. 6B). In contrast, ML-7 pretreatment blocked neutrophil-mediated internalization of claudin-1 and disruption of intercellular tight junctions, and also prevented stress fiber and intercellular gap formation (Fig. 6C). In addition, claudin-1 and perijunctional F-actin co-localization was restored at cell-cell junctions (Fig. 6C), displaying the characteristic ring-like structure similar to control epithelial cell monolayers.

\section{DISCUSSION}

Burn injury is responsible for around 40,000 hospitalizations and 4,000 deaths each year in the United States, despite tremendous advances in burn treatment and care in recent decades. Multiple organ failure accounts for $65 \%$ of these deaths, and infection is associated with $46 \%$ (30). Systemic inflammation and burn wound infections represent a major cause of mortality in patients with severe burns (9). Increased intestinal paracellular permeability and bacterial translocation during thermal injury also contribute to the development of SIRS and MOF. Given that gut barrier dysfunction occurs shortly after burn, it may play a more active and pivotal role in the pathogenesis of burn-related inflammatory syndromes than previously anticipated. A better understanding of the mechanisms underlying gut barrier impairment may help refine therapeutic strategies and improve outcomes for burned patients. In the present study, we demonstrate that nmMLCK directly mediates leukocyte adhesion and infiltration into gut tissue, and actively regulates intestinal epithelial barrier properties during thermal injury. Genetic deletion of nmMLCK not only confers protection against burn-induced intestinal epithelial paracellular hyperpermeability and mucosal villous damage in vivo, but also suppresses leukocyte activation, adhesion, and infiltration into intestinal villous lamina propria. These studies provide compelling evidence that nmMLCK 
regulates both intestinal mucosal barrier function and inflammatory response in burn injury. Notably, the current findings are in agreement with previous reports that epithelial nmMLCK plays a vital role in intestinal epithelial barrier dysfunction induced by cytokines (TNFa, interferon- $\gamma$, interleukin-1 $\beta)(24,25,31)$, activated T cells (26), and gastrointestinal infections (reviewed by 1 ).

The functional importance of neutrophil recruitment and extravasation in intestinal barrier dysfunction in burns has been well documented $(4,11,15)$. Indeed, the present data revealed that thermal injury led to PMN activation and recruitment in mesenteric postcapillary venules, accompanied by PMN infiltration into intestinal lamina propria and subsequent mucosal villous injury. In contrast, these inflammatory responses were largely prevented by genetic deletion of nmMLCK. In addition, depletion of mouse PMN with a neutralizing antibody diminished burn-induced intestinal hyperpermeability to a similar extent as nmMLCK deficiency. This further supports the vital role of nmMLCK and PMN activation/ transmigration in gut inflammation and barrier loss during thermal injury. Of note, burns induced severe leukocyte infiltration into intestinal lamina propria in WT mice after $4 \mathrm{~h}$, whereas gut injury was intermediate-to-severe at this time point. This apparent discrepancy suggests that gut leukocyte recruitment precedes intestinal epithelial barrier dysfunction and mucosal impairment, which is most likely more evident at later time points post-burn.

nmMLCK is expressed in both endothelial cells and leukocytes, both of which are actively involved in leukocyte adhesion and transvascular migration in acute inflammation. However, the mechanisms by which nmMLCK regulates PMN recruitment remain only partially understood. On the one hand, nmMLCK was recently reported to mediate neutrophil activation and transendothelial migration in septic lung inflammation through activation of $\beta 2$ integrin and the tyrosine kinase Pyk2 (22). On the other hand, endothelial nmMLCK could be activated to trigger microvascular endothelial actin-myosin contraction and hyperpermeability during vascular inflammation (18), which in turn promotes PMN recruitment and transvascular migration. Although the relative contribution of neutrophil and endothelial nmMLCK to leukocyte recruitment and intestinal mucosal injury in burns remains elusive, it seems probable that both leukocytic and endothelial nmMLCK are key in the pathophysiology. Indeed the highly concerted leukocyte transmigration requires both leukocyte activation/adhesion and intercellular junction reorganization in inflamed microvascular endothelium. In support of this conclusion, MLCK has been shown to mediate the endothelial actomyosin contractile response and microvascular leakage in burns and vascular inflammation $(5,18,23)$, which in turn augments leukocyte recruitment and transvascular migration. Moreover, nmMLCK deficiency enhances endothelial barrier function and prevents burn-induced microvascular hyperpermeability (6), which supports a regulatory role of endothelial nmMLCK in vascular inflammation. In this study, we demonstrate that burn-induced intestinal leukocyte recruitment is significantly diminished and gut barrier injury and mucosal villous damage prevented in nmMLCK-/- mice, suggesting that endothelial, epithelial, and leukocytic nmMLCK all contribute to the burninduced intestinal inflammatory response and gut barrier loss. Thus nmMLCK likely plays a critical role in PMN-dependent intestinal microvascular and mucosal epithelial barrier dysfunction in burns and other inflammatory injuries.

Neutrophil adhesion and infiltration into villous lamina propria and subepithelial interstitium provide an important line of defense against invading microbial pathogens. However, excessive release of toxic oxygen radical species, proteolytic enzymes and cytokines (e.g. interleukin-8) by activated PMNs in traumatic and thermal injuries often causes intestinal epithelial barrier dysfunction and potent gut inflammation $(11,12,15,32)$, exacerbating subsequent bacterial translocation and the development of septic complications. Meanwhile, inflammatory mediator-activated intestinal endothelial and epithelial cells express and 
release a range of chemoattractants such as chemokines and cytokines, augmenting leukocyte recruitment and extravasation (19). Intestinal epithelial barrier property is chiefly determined by the integrity of the intercellular tight junctions between adjacent epithelial cells (reviewed by 1,19 ). In this study, we demonstrate that activated neutrophils led to intestinal epithelial monolayer barrier dysfunction in a time-dependent pattern, coupled with MLCK activation and MLC phosphorylation. Moreover, claudin-1, an important epithelial TJ structural protein, was sequestrated from apical TJs and internalized into subapical intracellular compartments after PMN stimulation, indicating the disruption or disassembly of intestinal inter-epithelial junctions. In addition, the intestinal epithelial barrier breakdown is further corroborated by stress fiber production, intercellular gap formation, and disrupted co-localization of claudin-1 with peri-junctional F-actin. These intestinal epithelial responses evoked by neutrophils were prevented by pharmacologic inhibition of MLCK, emphasizing the critical signaling role of nmMLCK in the mediation of PMN-dependent intestinal epithelial actin-myosin ring contraction and barrier disruption in traumatic and other inflammatory injuries. Altered epithelial claudin-1 expression patterns have been associated with gut epithelial barrier dysfunction in infectious bowl disease (1), suggesting that claudin-1 may be an attractive cell marker for epithelial TJ integrity and a possible downstream regulatory effector of nmMLCK signaling. It is obvious that disruption of epithelial TJs causes intestinal hyperpermeability, and restoration of TJ structure promotes epithelial barrier property.

In conclusion, this study provides direct evidence that thermal injury causes intestinal barrier dysfunction and mucosal villous injury characterized by increased paracellular leak and neutrophil infiltration. Genetic deletion of nmMLCK inhibits neutrophil adhesion and infiltration into tissues and diminishes intestinal paracellular hyperpermeability in thermal injury. nmMLCK likely plays a prominent role in the pathophysiological regulation of intestinal barrier function. These findings may help develop more effective therapeutic strategies for treatment of trauma, burns and other related inflammatory injuries.

\section{Acknowledgments}

\section{Sources of Funding}

This work was supported by VA Merit Review 5101BX000799, the NIH grants HL96640, HL61507, and HL70752.

\section{References}

1. Turner JR. Intestinal mucosal barrier function in health and disease. Nat Rev Immunol. 2009; 9(11): 799-809. [PubMed: 19855405]

2. Gosain A, Gamelli RL. Role of the gastrointestinal tract in burn sepsis. J Burn Care Rehabil. 2005; 26(1):85-91. [PubMed: 15640741]

3. Demling RH. The burn edema process: current concepts. J Burn Care Rehabil. 2005; 26(3):207227. [PubMed: 15879742]

4. Magnotti LJ, Deitch EA. Burns, bacterial translocation, gut barrier function, and failure. J Burn Care Rehabil. 2005; 26(5):383-391. [PubMed: 16151282]

5. Huang Q, Xu W, Ustinova E, Wu M, Childs E, Hunter F, Yuan SY. Myosin light chain kinasedependent microvascular hyperpermeability in thermal injury. Shock. 2003; 20(4):363-368. [PubMed: 14501951]

6. Reynoso R, Perrin RM, Breslin JW, Daines DA, Watson KD, Watterson DM, Wu MH, Yuan S. A role for long chain myosin light chain kinase (MLCK-210) in microvascular hyperpermeability during severe burns. Shock. 2007; 28(5):589-595. [PubMed: 17577141] 
7. Breslin JW, Wu MH, Guo M, Reynoso R, Yuan SY. Toll-like receptor 4 contributes to microvascular inflammation and barrier dysfunction in thermal injury. Shock. 2008; 29(3):349-355. [PubMed: 17704733]

8. Costantini TW, Loomis WH, Putnam JG, Drusinsky D, Deree J, Choi S, Wolf P, Baird A, Eliceiri B, Bansal V, Coimbra R. Burn-induced gut barrier injury is attenuated by phosphodiesterase inhibition: effects on tight junction structural proteins. Shock. 2009; 31(4):416-422. [PubMed: 18791495]

9. Church D, Elsayed S, Reid O, Winston B, Lindsay R. Burn wound infections. Clin Microbiol Rev. 2006; 19(2):403-434. [PubMed: 16614255]

10. Horton JW. Bacterial translocation after burn injury: the contribution of ischemia and permeability changes. Shock. 1994; 1(4):286-290. [PubMed: 7735963]

11. Al-Ghoul WM, Khan M, Fazal N, Sayeed MM. Mechanisms of postburn intestinal barrier dysfunction in the rat: roles of epithelial cell renewal, E-cadherin, and neutrophil extravasation. Crit Care Med. 2004; 32(8):1730-1739. [PubMed: 15286551]

12. Deitch EA. Intestinal permeability is increased in burn patients shortly after injury. Surgery. 1990; 107(4):411-416. [PubMed: 2108508]

13. Chen LW, Chen PH, Chang WJ, Wang JS, Karin M, Hsu CM. IкB-kinase/nuclear factor- $\kappa B$ signaling prevents thermal injury-induced gut damage by inhibiting c-Jun NH2-terminal kinase activation. Crit Care Med. 2007; 35(5):1332-1340. [PubMed: 17414734]

14. Eaves-Pyles T, Alexander JW. Rapid and prolonged impairment of gut barrier function after thermal injury in mice. Shock. 1998; 9(2):95-100. [PubMed: 9488253]

15. Sir O, Fazal N, Choudhry MA, Gamelli RL, Sayeed MM. Neutrophil depletion prevents intestinal mucosal permeability alterations in burn-injured rats. Am J Physiol Regul Integr Comp Physiol. 2000; 278(5):R1224-R1231. [PubMed: 10801291]

16. Goto M, Samonte V, Ravindranath T, Sayeed MM, Gamelli RL. Burn injury exacerbates hemodynamic and metabolic responses in rats with polymicrobial sepsis. J Burn Care Res. 2006; 27(1):50-59. [PubMed: 16566537]

17. Tinsley JH, Wu MH, Ma W, Taulman AC, Yuan SY. Activated neutrophils induce hyperpermeability and phosphorylation of adherens junction proteins in coronary venular endothelial cells. J Biol Chem. 1999; 274(35):24930-24934. [PubMed: 10455168]

18. Yuan SY, Wu MH, Ustinova EE, Guo M, Tinsley JH, De Lanerolle P, Xu W. Myosin light chain phosphorylation in neutrophil-stimulated coronary microvascular leakage. Circ Res. 2002; 90(11): 1214-1221. [PubMed: 12065325]

19. Chin AC, Parkos CA. Pathobiology of neutrophil transepithelial migration: implications in mediating epithelial injury. Annu Rev Pathol. 2007; 2:111-143. [PubMed: 18039095]

20. Verin AD, Lazar V, Torry RJ, Labarrere CA, Patterson CE, Garcia JG. Expression of a novel high molecular-weight myosin light chain kinase in endothelium. Am J Respir Cell Mol Biol. 1998; 19:758-766. [PubMed: 9806740]

21. Clayburgh DR, Rosen S, Witkowski ED, Wang F, Blair S, Dudek S, Garcia JG, Alverdy JC, Turner JR. A differentiation-dependent splice variant of myosin light chain kinase, MLCK1, regulates epithelial tight junction permeability. J Biol Chem. 2004; 279(53):55506-55513. [PubMed: 15507455]

22. Xu J, Gao XP, Ramchandran R, Zhao YY, Vogel SM, Malik AB. Nonmuscle myosin light-chain kinase mediates neutrophil transmigration in sepsis-induced lung inflammation by activating $\beta 2$ integrins. Nat Immunol. 2008; 9:880-886. [PubMed: 18587400]

23. Tinsley JH, Teasdale NR, Yuan SY. Myosin light chain phosphorylation and pulmonary endothelial cell hyperpermeability in burns. Am J Physiol Lung Cell Mol Physiol. 2004; 286(4):L841-L847. [PubMed: 14672924]

24. Zolotarevsky Y, Hecht G, Koutsouris A, Gonzalez DE, Quan C, Tom J, Mrsny RJ, Turner JR. A membrane-permeant peptide that inhibits MLC kinase restores barrier function in in vitro models of intestinal disease. Gastroenterology. 2002; 123(1):163-172. [PubMed: 12105845]

25. Ma TY, Boivin MA, Ye D, Pedram A, Said HM. Mechanism of TNF-a modulation of Caco-2 intestinal epithelial tight junction barrier: role of myosin light-chain kinase protein expression. Am J Physiol Gastrointest Liver Physiol. 2005; 288(3):G422-G430. [PubMed: 15701621] 
26. Clayburgh DR, Barrett TA, Tang Y, Meddings JB, Van Eldik LJ, Watterson DM, Clarke LL, Mrsny RJ, Turner JR. Epithelial myosin light chain kinase-dependent barrier dysfunction mediates $\mathrm{T}$ cell activation-induced diarrhea in vivo. J Clin Invest. 2005; 115(10):2702-2715. [PubMed: 16184195]

27. Korompai FL, Yuan SY. Ventral burn in rats: an experimental model for intravital microscopic study of microcirculation. Burns. 2001; 1386:1-7.

28. Costantini TW, Bansal V, Krzyzaniak M, Putnam JG, Peterson CY, Loomis WH, Wolf P, Baird A, Eliceiri BP, Coimbra R. Vagal nerve stimulation protects against burn-induced intestinal injury through activation of enteric glia cells. Am J Physiol Gastrointest Liver Physiol. 2010; 299(6):G1308-1318. [PubMed: 20705905]

29. Hidalgo IJ, Raub TJ, Borchardt RT. Characterization of the human colon carcinoma cell line (Caco-2) as a model system for intestinal epithelial permeability. Gastroenterology. 1989; 96(3): 736-749. [PubMed: 2914637]

30. Bloemsma GC, Dokter J, Boxma H, Oen IM. Mortality and causes of death in a burn centre. Burns. 2008; 34(8):1103-1107. [PubMed: 18538932]

31. Wang F, Graham WV, Wang Y, Witkowski ED, Schwarz BT, Turner JR. Interferon- $\gamma$ and tumor necrosis factor-a synergize to induce intestinal epithelial barrier dysfunction by up-regulating myosin light chain kinase expression. Am J Pathol. 2005; 166(2):409-419. [PubMed: 15681825]

32. Akhtar S, Li X, Chaudry IH, Choudhry MA. Neutrophil chemokines and their role in IL-18mediated increase in neutrophil O2- production and intestinal edema following alcohol intoxication and burn injury. Am J Physiol Gastrointest Liver Physiol. 2009; 297(2):G340-G347. [PubMed: 19497959] 
A

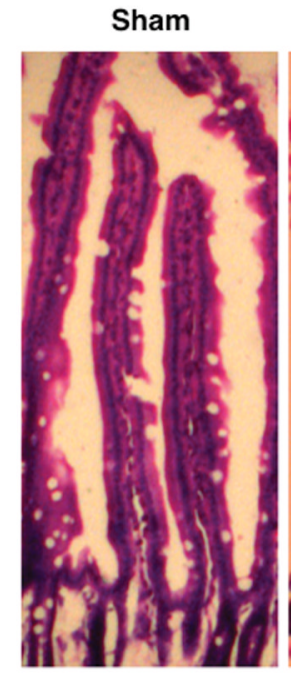

B

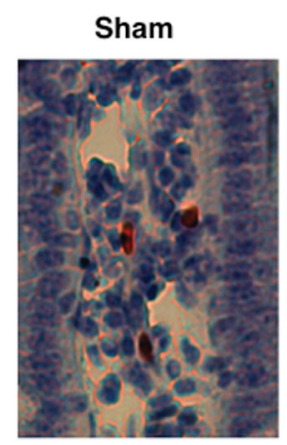

C

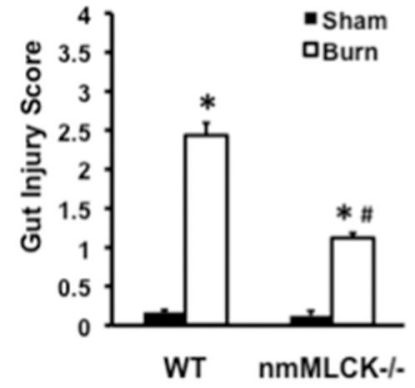

WT, Burn

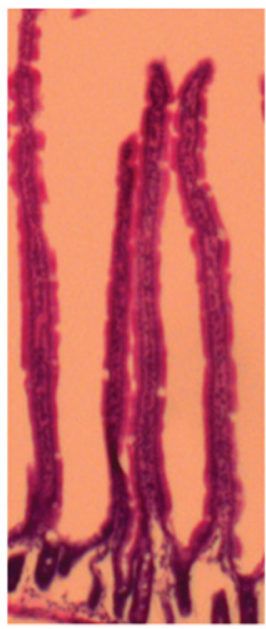

nmMLCK-/-, Burn

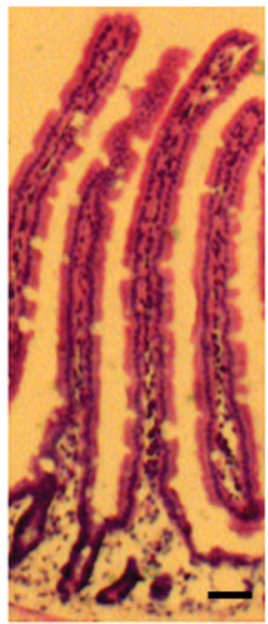

WT, Burn

nmMLCK-/-, Burn
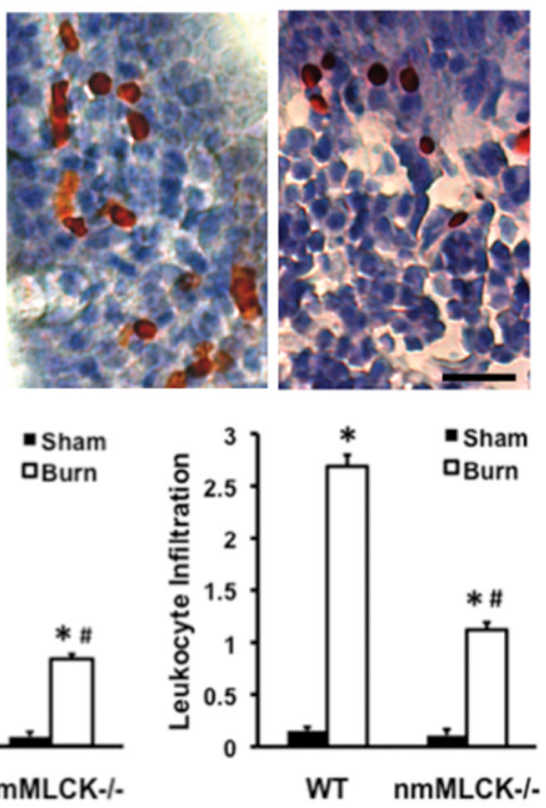

Figure 1.

Burn induces gut mucosal barrier disruption and leukocyte infiltration into the lamina propria of small intestines. Segments of distal small intestines were harvested $4 \mathrm{~h}$ post-burn, and cryosections of gut tissues were stained with hematoxylin and eosin. A, Microscopic examination of the small intestinal mucosa revealed marked villous thinning, shrinkage, and disorganization in WT mice subjected to thermal injury, and such gut damage was prevented in nmMLCK-deficient mice receiving burns. Scare bar $=100 \mu \mathrm{m}$. B, Immunohistochemical staining to illustrate the infiltration of leukocytes into small intestinal lamina propria of WT mice with thermal injury. The number of labeled leukocytes (brown) was decreased in nmMLCK-deficient mice compared with wild type mice after thermal injury. Brown staining in the lamina propria indicates the presence of neutrophils. Scale bar $=20 \mu \mathrm{m}$. C, Intestinal injury was scored using a pathological scoring system with a scale of 0 (normal) to 4 (severe), and is displayed in the left bar graph. Leukocyte infiltration into intestinal lamina 
propria was scored on a scale of 0 (0-5 PMNs) to 3 (21 PMNs, and above), and is depicted in the right bar graph. ${ }^{*} P<0.05$ vs. sham WT, and ${ }^{\#} P<0.01$ vs. burn WT. 
A

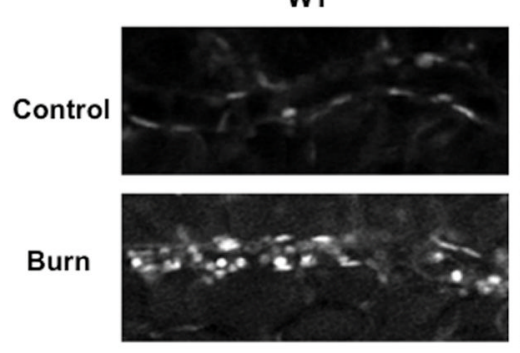

B

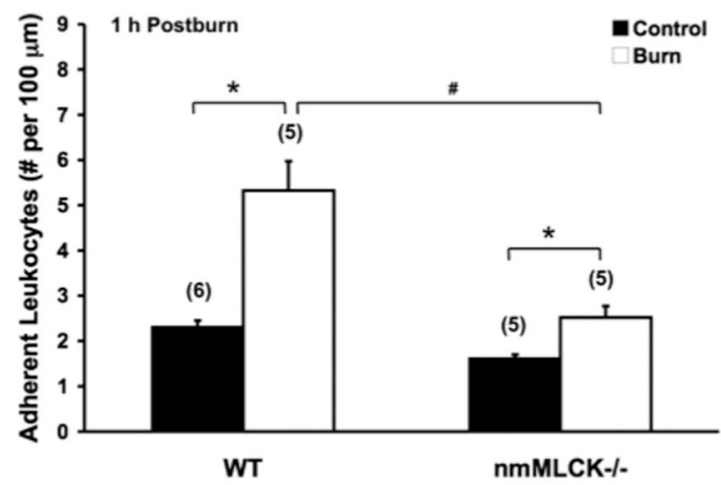

C

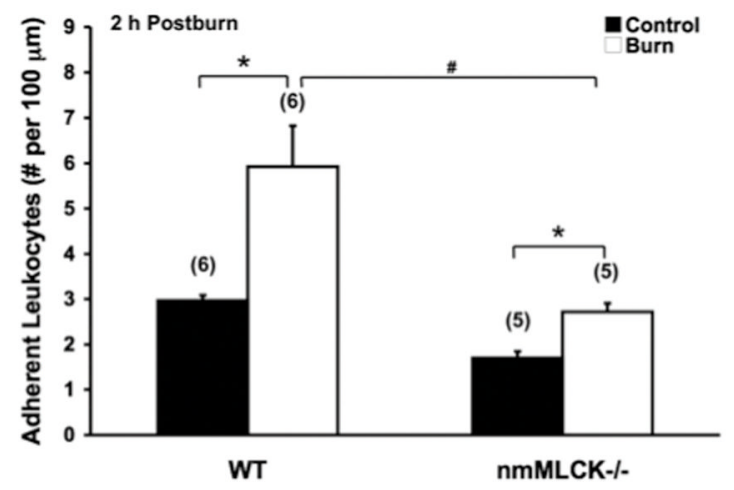

Figure 2.

Leukocyte adhesion in mesenteric postcapillary venules of WT and nmMLCK-deficient mice subjected to sham or burn. A, Representative intravital microscopic images of postcapillary venules (15-25 $\mu \mathrm{m}$ in diameter) at $2 \mathrm{~h}$ after burn. B, Thermal injury induces significant increases in leukocyte adhesion to endothelium in mesenteric postcapillary venules of WT mice; by contrast, such leukocyte recruitment was significantly attenuated in nmMLCK-/- mice that received thermal injury. ${ }^{*} P<0.05$ and ${ }^{\#} P<0.01$ for the indicated groups. 


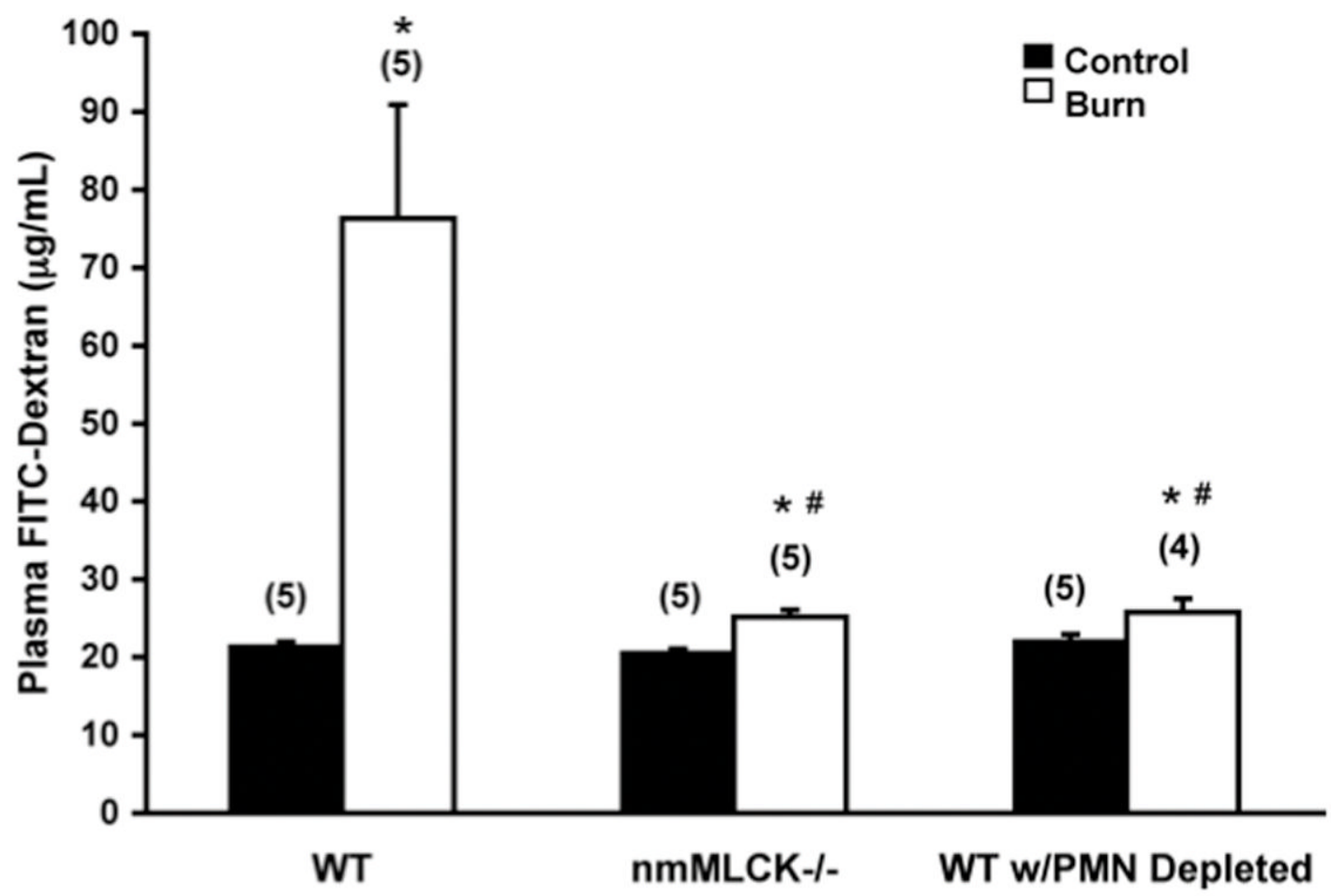

Figure 3.

Both nmMLCK deficiency and PMN depletion prevented burn-induced intestinal hyperpermeability in vivo. Both WT and nmMLCK-deficient mice were subjected to thermal injury, and small intestinal permeability to FITC-dextran $(4.4 \mathrm{kDa})$ was determined by measuring plasma tracer concentrations $4 \mathrm{~h}$ post-burn with a fluorescence microplate reader. Intestinal permeability was significantly increased in WT mice post-burn, and the response was abolished in the absence of nmMLCK or with PMN depletion. $* P<0.05$ vs. control; ${ }^{\#} P<0.01$ vs. WT with burn. 
A

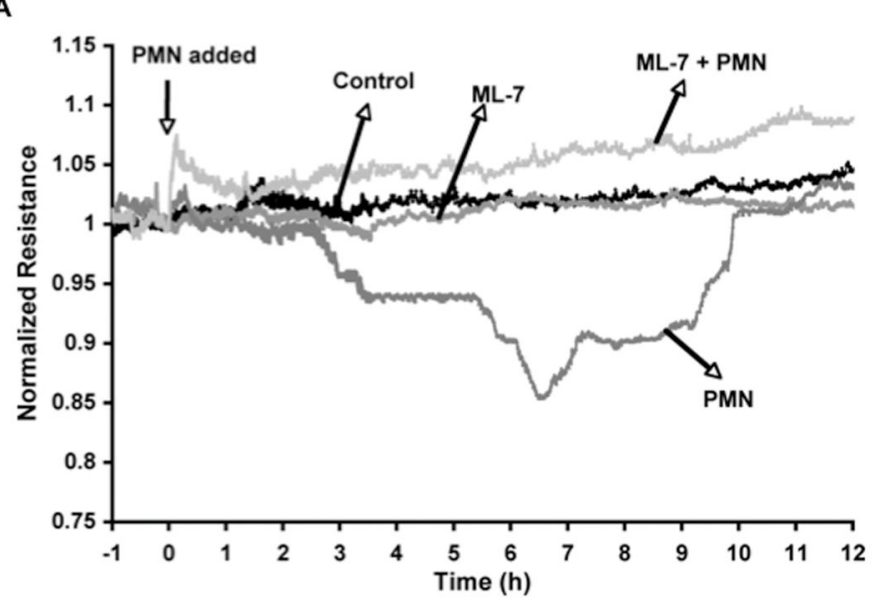

B

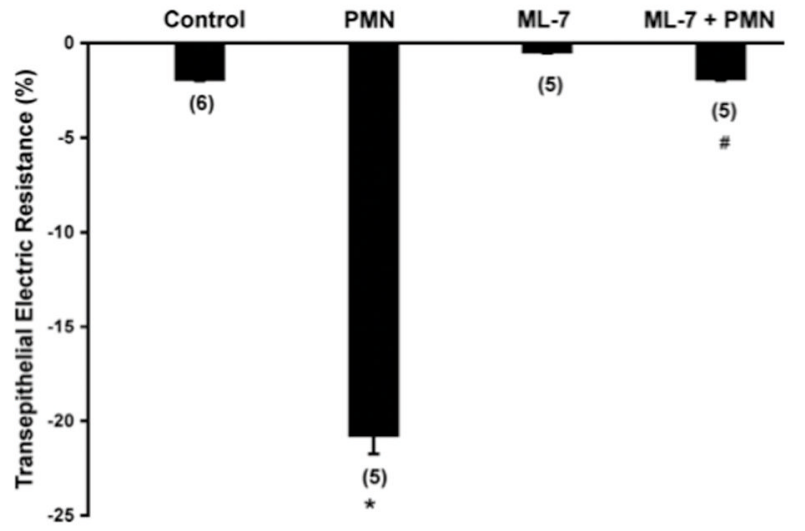

Figure 4.

Activated neutrophils induced intestinal epithelial barrier dysfunction in Caco-2 cell monolayers. A, Time courses of epithelial TER changes induced by fMLP-activated neutrophils and the inhibitory effect of ML-7, a selective MLCK inhibitor, on TER decline. Caco-2 (human epithelial colon adenocarcinoma) cells were pretreated with $1 \mu \mathrm{M}$ ML-7 for $30 \mathrm{~min}$, followed by stimulation with fMLP-activated neutrophils. Shown are typical tracings of TER normalized to the baseline immediately prior to neutrophil stimulation. B, Activated neutrophil-induced epithelial barrier dysfunction is prevented by ML-7. Shown are the maximal changes $(6-8 \mathrm{hr})$ in TER. $* P<0.01$ vs. vehicle control; $\# P<0.01$ vs. PMN alone. 
A

pMLC

(S19/T18)
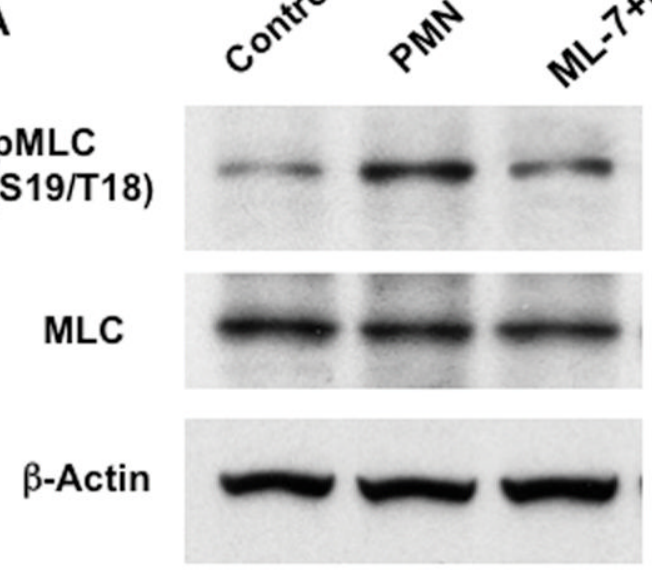

(1)
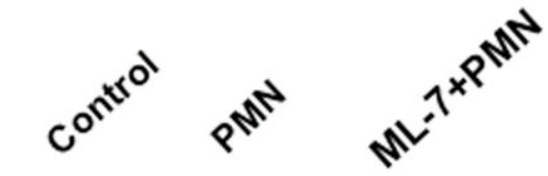

\section{응}

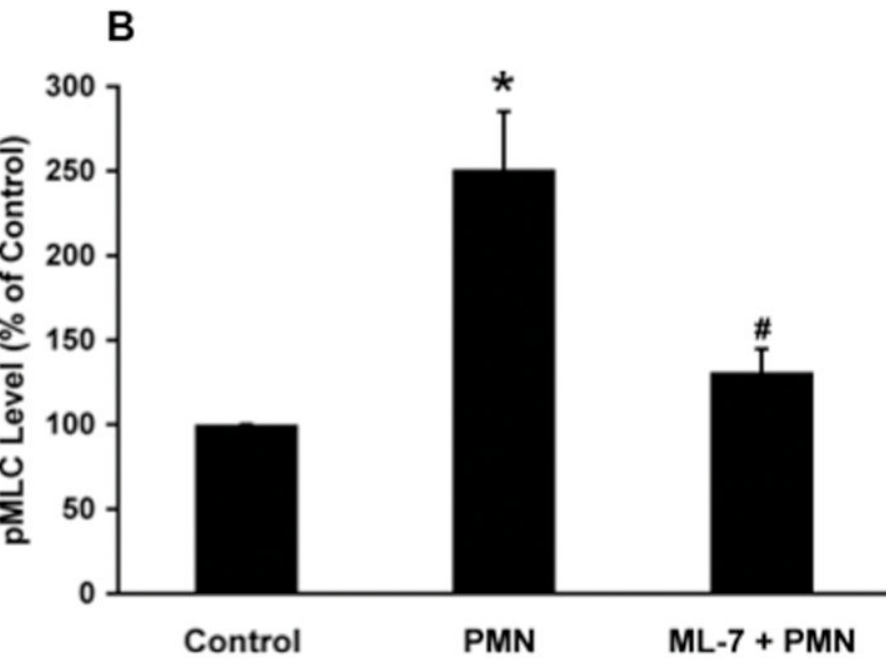

Figure 5.

Neutrophils induced MLCK-dependent MLC phosphorylation in Caco-2 cells. A, Activated neutrophils induced an increase in MLC phosphorylation, which was attenuated by pretreatment with $1 \mu \mathrm{M}$ ML-7. Shown are representative images of 3 separate experiments (6 hr after PMN) indicating levels of MLC (Thr18/Ser18) phosphorylation, total MLC and $\beta$-actin in Caco- 2 cells. B, Quantification of levels of phosphorylated MLC in Caco-2 cells treated with vehicle control, activated neutrophils, and ML-7 plus neutrophils, respectively. 

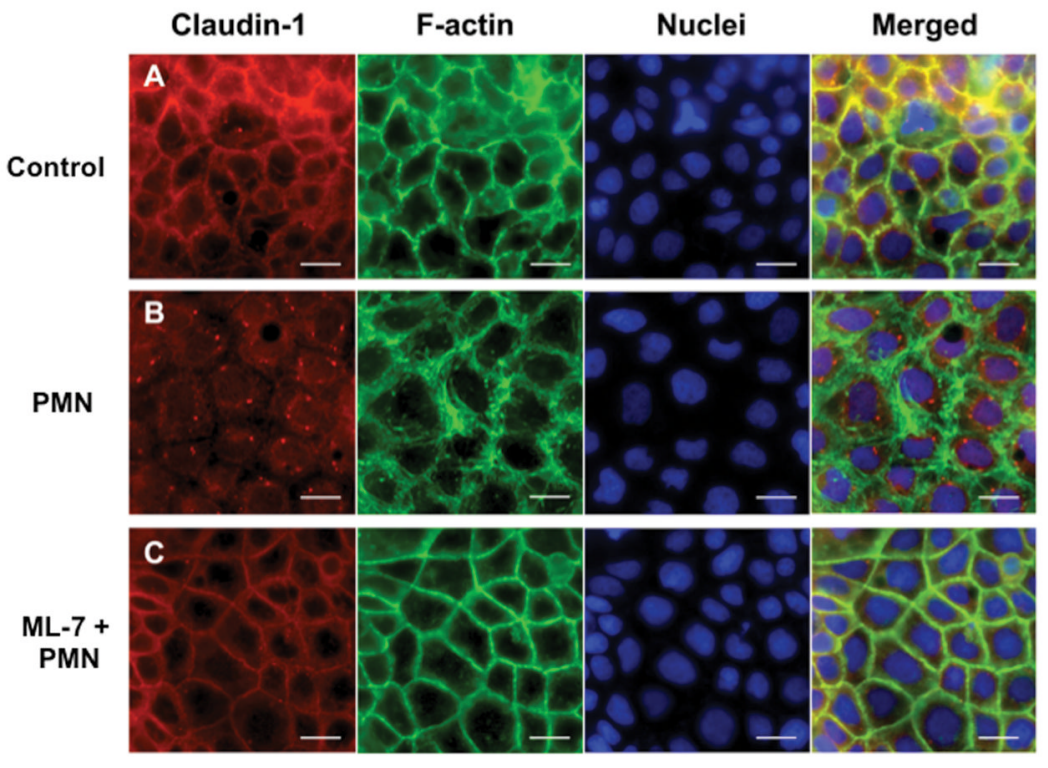

Figure 6.

Activated neutrophils induced claudin-1 re-distribution and intercellular gap formation in intestinal epithelial cells. Caco-2 cell monolayers were treated with vehicle, fMLP-activated neutrophils, and neutrophils plus pre-treatment with $1 \mu \mathrm{M}$ ML-7, respectively. A, Tight junction molecule claudin-1 and F-actin were largely localized at intact cell-cell junctions under normal conditions (vehicle control), and the merge field shows their co-localization, which is indicative of normal intestinal epithelial integrity. B, After neutrophil stimulation, claudin-1 was sequestrated from intercellular junctions and internalized into subapical intracellular compartments in Caco-2 cells, in concomitance with stress fiber production and inter-cellular gap formation. The disrupted co-localization of claudin-1 with F-actin was indicative of the disassembly of epithelial tight junctions (the merge field). C, Pre-treatment of Caco-2 cells with $1 \mu \mathrm{M}$ ML-7, a selective MLCK inhibitor, suppressed neutrophilinduced stress fiber formation, restored the intercellular junction distribution and colocalization of claudin-1 and F-actin, and rescued epithelial integrity disrupted by neutrophils (the merge field). Bar $=20 \mu \mathrm{m}$. 